\title{
Crystals in the Void
}

\author{
Senya Shlosman ${ }^{\natural, \sharp, b}$ \\ ${ }^{\natural}$ Skolkovo Institute of Science and Technology, Moscow, Russia; \\ \#Aix Marseille University, University of Toulon, \\ CNRS, CPT, Marseille, France; \\ ${ }^{b}$ Inst. of the Information Transmission Problems, \\ RAS, Moscow, Russia. \\ Senya.Shlosman@cpt.univ-mrs.fr, shlos@iitp.ru
}

October 6, 2018

\begin{abstract}
We study the problem of the crystal formation in vacuum.
\end{abstract}

\section{Introduction}

By now, the rigorous theory of the Wulff shapes is well developed in statistical mechanics. It describes the asymptotic shape of a large (random) droplet of one phase surrounded by the sea of coexisting different phase. Such a large droplet arises if forced into a system at the phase coexistence by the canonical constraint (see [DKS, $\mathrm{B}, \mathrm{CP}$ ), or else when it is created spontaneously during the process of dynamical relaxation from the metastable state to the stable one $([\mathrm{SS}])$. All the papers cited are dealing with the Ising model at zero

Part of this work has been carried out in the framework of the Labex Archimede (ANR-11-LABX-0033) and of the A*MIDEX project (ANR-11-IDEX-0001-02), funded by the "Investissements d'Avenir" French Government programme managed by the French National Research Agency (ANR). Part of this work has been carried out at IITP RAS. The support of Russian Foundation for Sciences (project No. 14-50-00150) is gratefully acknowledged. 
magnetic field and the temperature below the critical one, i.e. when there is the phase coexistence phenomenon.

In $[\mathrm{R}]$ a different question is asked: suppose a certain given amount of matter is put into void space (for example, this matter can be described by the Ising model at a positive magnetic field, in which regime there is no phase coexistence). Which shape this matter will assume? Alternatively, consider a droplet of water, floating in oil, and then low the temperature so that the water freezes. What will be the shape of the ice crystal one sees?

In the present note we provide rigorous answers to this question for some models, which in some cases are different from the answers anticipated in $[\mathrm{R}]$. We also formulate conjectures in situations where rigorous answers are not available currently.

\section{Lattice case}

\subsection{Ising crystal in the void}

Consider the Ising \pm 1 spins $\sigma$ in $d$ dimensions under magnetic field $h>0$ at temperature $\beta^{-1}$, given by the Hamiltonian

$$
H^{I}(\sigma)=-\sum_{x, y \text { n.n. }} \sigma_{x} \sigma_{y}-h \sum_{x} \sigma_{x}, \quad x, y \in \mathbb{Z}^{d} .
$$

The idea in $[\mathrm{R}]$ is to consider the finite container $V$ as a third parameter of the model. Namely, let $Z(\beta, h, V)$ denote the partition function in $V$ with free boundary condition. Denote by $\mathbb{T}_{N}^{d}$ the $d$-dimensional torus of volume $N^{d}$. The void crystal model is defined by the partition function

$$
\Xi(\beta, h, N, \mathbf{f})=\sum_{\substack{V \subset \mathbb{T}_{N}^{d}: \\ \operatorname{vol}(V)=\left\lfloor\mathbf{f} N^{d}\right\rfloor}} Z(\beta, h, V) .
$$

Here $\mathbf{f}>0$ is the number defining the proportion of the box $\mathbb{T}_{N}^{d}$ filled by the Ising matter. We take $\mathbf{f}$ to be small enough, so that the shape of the box $\mathbb{T}_{N}^{d}$ will have no effect on the typical $V$-s.

The corresponding probability distribution $\mathbf{P}_{N}(*)$ on the boxes $V \subset \mathbb{T}_{N}^{d}$ with $\operatorname{vol}(V)=\left\lfloor\mathbf{f} N^{d}\right\rfloor$ is given by

$$
\mathbf{P}_{N}(V)=\frac{Z(\beta, h, V)}{\Xi(\beta, h, N, \mathbf{f})}
$$


This probability distribution is somewhat different from the one suggested in $[\mathrm{R}]$ : we do not restrict $V$ to be star-shaped, nor, in fact, that $V$ is connected or simply-connected. This generality is physically more reasonable.

The question we want to study is the typical properties of the shapes $V$ as the size $N$ of our system goes to $\infty$. To this end we first reformulate the question as a question about the behavior of a different model at the phase coexistence.

The new model is again a lattice spin model $\eta$ on $\mathbb{Z}^{d}$, taking now values $+1,0,-1$, and defined by the Hamiltonian

$$
H(\eta)=-\sum_{x, y \text { n.n. }} \eta_{x} \eta_{y}-h \sum_{x} \eta_{x}-[d+k] \sum_{x}\left(1-\eta_{x}^{2}\right) .
$$

In words, we put our \pm 1 Ising model into the ideal gas of non-interacting 0 spins, which are subject to the magnetic field of strength $[d+k]$. Note that the question of the behavior of the random boxes $V$ of size $|V|=\left\lfloor\mathbf{f} N^{d}\right\rfloor$ under the distribution (2) is equivalent to the question of the behavior of the random boxes $W(\eta) \equiv\left\{x \in \mathbb{T}_{N}^{d}: \eta_{x} \neq 0\right\}$ of the model (3), considered

- at the temperature $\beta^{-1}$,

- in the same magnetic field $h>0$,

- under 'canonical' constraint $\sum_{x}\left(1-\eta_{x}^{2}\right)=(1-\mathbf{f}) N^{d}$,

- for any value of the magnetic field $[d+k]$, acting on the 0 spins.

Consider the translation-invariant ground state configurations of the Hamiltonian (3). For $k<h$ it is the configuration $\eta^{+} \equiv+1$, for $k<h$ it is the configuration $\eta^{0} \equiv 0$, while for $k=h$ the Hamiltonian (3) is degenerate and has two ground state configurations: $\eta=+1$ and $\eta=0$. Moreover, it is easy to check that at $k=h$ the Peierls stability condition holds:

Consider a finite box $V \subset \mathbb{Z}^{d}$, and define the configuration $\eta^{V}$ by

$$
\eta_{x}^{V}=\left\{\begin{array}{cc}
+1 & \text { if } x \in V \\
0 & \text { otherwise }
\end{array}\right.
$$

Then for some $\tau>0$ we have

$$
H\left(\eta^{V}\right)-H\left(\eta^{0}\right) \geq \tau|\partial V|,
$$


where $\partial V=\left\{\{x, y\}: x, y\right.$ n.n. $\left.\in \mathbb{Z}^{d}, x \in V, y \notin V\right\}$. (In fact, $H\left(\eta^{V}\right)-H\left(\eta^{0}\right)=$ $|\partial V|$, so the Peierls constant $\tau$ can be taken to be 1.)

Therefore the Pirogov-Sinai theory, see e.g. [S], applies to our model (3) . It claims that for any $d \geq 2$ there exists a value $\beta_{d}<\infty$ such that for all $\beta>\beta_{d}$ there exist the value $k(\beta, h)$ of the magnetic field $k$ at which there are two translation-invariant Gibbs states corresponding to the Hamiltonian (3) ; one is small perturbation of the configuration $\eta^{+}$, while the other - of the configuration $\eta^{0}$. Moreover, $k(\beta, h) \rightarrow h$, as $\beta \rightarrow \infty$. (In fact, $k(\beta, h)$ can be easily expressed via the free energy $f(\beta, h)$ of the Ising model.)

Summarizing, we see that the study 'in the void' - of the behavior of the random box $V$ under the distribution $\mathbf{P}_{N}(V)$ above, is equivalent to the study of the random box $W$ at the same temperature $\beta$ and the field $k=k(\beta, h)$, i.e. at coexistence (provided $\beta$ is large).

In particular, for $d=2$ all the machinery and all the results of [DKS] are valid in our situation, provided $\beta$ is large enough:

Theorem 1 Let $h>0, \beta>\beta_{d=2}$ and $0<\mathbf{f}<\frac{1}{10}$. There exists the subset $\mathcal{V}_{N}$ of boxes, $\mathcal{V}_{N}=\left\{V \subset \mathbb{T}_{N}^{2}: \operatorname{vol}(V)=\mathbf{f} N^{2}\right\}$, such that $\mathbf{P}_{N}\left(\mathcal{V}_{N}\right) \rightarrow 1$ as $N \rightarrow \infty$, which has the following properties:

1. Among the connected components $\partial_{i} V$ of the boundary $\partial V$ of a box $V \in \mathcal{V}_{N}$ there exists exactly one - say, $\partial_{1} V \equiv \Gamma \subset \partial V$ - which is 'big': $\operatorname{diam}\left(\partial_{1} V\right) \sim N$; all other components are 'small': $\operatorname{diam}\left(\partial_{i} V\right) \sim \ln N, i=$ $2,3, \ldots$

2. The contour $\Gamma$ has asymptotic shape $W$, with

$$
W=W(\beta, h) \subset \mathbb{R}^{2}
$$

being some smooth $\left(\mathcal{C}^{\infty}\right)$ strictly convex centrally symmetric closed curve. More precisely, for every $V \in \mathcal{V}_{N}$ and its big boundary component $\Gamma=\partial_{1} V$ there exists a vector $x(\Gamma)$ such that the shifted contour, $\Gamma+x(\Gamma)$, satisfies

$$
\operatorname{dist}_{\mathbf{H}}(\Gamma+x(\Gamma), c N W) \leq N^{3 / 4} .
$$

Here dist $_{\mathbf{H}}$ is the Hausdorf distance, and the scaling factor $c$ depends on $\beta, h$ and $\mathbf{f}$ only.

3. The curve $W$ is the Wulff shape, corresponding to the Hamiltonian (3) at the temperature $\beta$ and magnetic fields $h, k(\beta, h)$. Its construction is explained below. 
The proof of this theorem follows essentially the same lines as that in the book [DKS]. The difference is that [DKS] treats the Ising model, while here we have a different one. But the analysis of the proof given in [DKS] shows that its technique applies also to any 2D model within the PirogovSinai class. The differences are only notational and technical, though they will result in the doubling of the length of the proof.

The situation in the 3D case is similar, though some details differ: instead of the distance dist $_{\mathbf{H}}$ one has to consider the $L^{1}$-distance, the exponent in (5) is not known (though $\ln N$ is expected, compare with $[\mathrm{K}]$ ), the Wulff surface $W$ is not strictly convex and is only $\mathcal{C}^{1}$, etc. For details, see $[\mathrm{B}, \mathrm{BIV}, \mathrm{CP}]$.

\subsection{The Wulff shape}

In this section we will explain how to construct the curve $W$ entering our theorem, see (44). This curve is a solution to the Wulff variational problem, stated below. The variational problem has as its input a certain surface tension function, $\tau_{H, \beta}(*)$, which is defined by the Hamiltonian (3) and the inverse temperature $\beta$, and which will be defined next.

\subsubsection{Wulff problem}

Wulff variational problem is formulated as follows. Let $\tau(\mathbf{n}), \mathbf{n} \in S^{d-1}$ be some continuous function on the unit sphere $S^{d-1} \subset \mathbb{R}^{d}$. We suppose that $\tau>0$, and that $\tau$ is even. For every closed compact (hyper)surface $M^{d-1} \subset \mathbb{R}^{d}$ we define its surface energy as

$$
\mathcal{W}_{\tau}(M)=\int_{M} \tau\left(\mathbf{n}_{s}\right) d s
$$

where $\mathbf{n}_{s}$ is the normal vector to $M$ at $s \in M$. The functional $\mathcal{W}_{\tau}(M)$ has the meaning of the surface energy of the $M$-shaped droplet. It is called the Wulff functional. Let $W_{\tau}$ be the surface which minimizes $\mathcal{W}_{\tau}(\cdot)$ over all the surfaces enclosing the unit volume. Such a minimizer does exist and is unique up to translation. It is called the Wulff shape.

The following is the geometric construction of $W_{\tau}$. Consider the set

$$
K_{\tau}=\left\{\mathbf{x} \in \mathbb{R}^{d}: \forall \mathbf{n} \in S^{d-1}(\mathbf{x}, \mathbf{n}) \leq \tau(\mathbf{n})\right\} .
$$

If we define the half-spaces

$$
L_{\tau, \mathbf{n}}=\left\{\mathbf{x} \in \mathbb{R}^{d}:(\mathbf{x}, \mathbf{n}) \leq \tau(\mathbf{n})\right\},
$$


then

$$
K_{\tau}=\cap_{\mathbf{n}} L_{\tau, \mathbf{n}} ;
$$

in particular, $K_{\tau}$ is convex. It turns out that

$$
W_{\tau}=\lambda_{\tau} \partial\left(K_{\tau}\right)
$$

where the dilatation factor $\lambda_{\tau}$ is defined by the normalization: $\operatorname{vol}\left(\lambda_{\tau} K_{\tau}\right)=$ 1. The relation (6) is called the Wulff construction.

\subsubsection{Surface tension}

In this subsection we specify the surface tension function $\tau=\tau_{H, \beta}(*)$, which has to be used in the construction above.

Let $\theta \in \mathbb{S}^{1}$ be a unit vector in $\mathbb{R}^{2}$. Let us define the spin configuration $\eta^{\theta}$ on $\mathbb{Z}^{2}$ by

$$
\eta_{x}^{\theta}=\left\{\begin{array}{cc}
+1 & \text { if }\langle x, \theta\rangle \geq 0 \\
0 & \text { otherwise }
\end{array}\right.
$$

and let us also put

$$
\eta_{x}^{+} \equiv+1, \quad \eta_{x}^{0} \equiv 0 .
$$

Let $B_{n} \subset \mathbb{Z}^{2}$ be a square box centered at the origin, with a side $2 n$. Consider the partition functions $Z\left(\beta, h, k, B_{n} ; \eta^{\theta}\right), Z\left(\beta, h, k, B_{n} ; \eta^{+}\right)$, and $Z\left(\beta, h, k, B_{n} ; \eta^{0}\right)$, which are computed in the box $B_{n}$ for the Hamiltonian (3) with boundary conditions $\eta^{\theta}, \eta^{+}$and $\eta^{0}$. The surface tension $\tau_{H, \beta}$ is defined as

$$
\tau_{H, \beta}(\theta)=\lim _{n \rightarrow \infty}-\frac{1}{\beta l(\theta, n)} \ln \frac{Z\left(\beta, h, k, B_{n} ; \eta^{\theta}\right)}{\sqrt{Z\left(\beta, h, k, B_{n} ; \eta^{+}\right) Z\left(\beta, h, k, B_{n} ; \eta^{0}\right)}},
$$

where $l(\theta, n)$ is the length of the segment, obtained by intersecting the line $L(\theta)=\left\{x \in \mathbb{R}^{2}:\langle x, \theta\rangle=0\right\}$ and the box $B_{n}$.

Theorem 2 Let the field $k=k(\beta, h)$. Then the limit (7) exists, is positive for $\beta$ large enough and is smooth in $\theta$. It also satisfies the 'triangle inequality' (see relation (2.2.2) in [DKS]).

As a result, the properties of the curve $W$, listed in the Theorem 1, follow, see again [DKS]. 


\section{Continuum case}

Here we consider the case of crystals in $\mathbb{R}^{d}$. Much less is known here rigorously.

We will treat point random fields, defined by the interaction $U(x, y)=$ $U(|x-y|)$, which is supposed to be superstable. For example, LennardJones potential, or the potential

$$
U(|x-y|)=\left\{\begin{array}{cc}
+\infty & \text { if }|x-y| \leq 1, \\
|x-y|-3 & \text { if } 1<|x-y|<3, \\
0 & \text { if }|x-y| \geq 3
\end{array}\right.
$$

of $[\mathbf{R}]$ or just the hard-core interaction will go. The weight $w_{\beta, z}(\mathbf{x})$ of a configuration $\mathbf{x}=\left\{x_{i} \in \mathbb{R}^{d}, i=1, \ldots, n\right\}$ is given by

$$
w_{\beta, z}(\mathbf{x})=z^{n} \exp \left\{-\beta \sum_{i<j} U\left(x_{i}, x_{j}\right)\right\} \text {. }
$$

The parameter $z>0$ is called activity.

For a finite box $V \subset \mathbb{R}^{d}$ the partition function with free boundary conditions is defined as

$$
Z(\beta, z, V) \equiv Z(\beta, z, V, \varnothing)=\sum_{n=0}^{\infty} \int_{V^{n}} w_{\beta, z}(\mathbf{x}) d \mathbf{x}
$$

Since there is no natural measure on the space of all boxes $V \subset \mathbb{T}_{N}^{d}$ (these notations refer now to the continuous case of $\mathbb{R}^{d}$ ), we will proceed via some discretization procedure, the effect of which vanishes in the thermodynamic limit. For every $N$ we consider the partition of the torus $\mathbb{T}_{N}^{d}$ into cubes of size $\frac{1}{N}$ - i.e. into $N^{2 d}$ cubes, and we call a box $V \subset \mathbb{T}_{N}^{d}$ an $N$-box iff $V$ is the union of these $\frac{1}{N}$ cubes. (An $N$-box need not to be connected.)

The probability distribution $\mathbf{P}_{N}(*)$ on the $N$-boxes $V$ of size $\left\lfloor\mathbf{f} N^{d}\right\rfloor$ that we want to consider now is given by

$$
\mathbf{P}_{N}(V)=\frac{Z(\beta, z, V)}{\Xi(\beta, z, N, \mathbf{f})},
$$

where the partition function

$$
\Xi(\beta, z, N, \mathbf{f})=\sum_{\substack{V \subset \mathbb{T}_{N}^{d}: \\ \operatorname{vol}(V)=\left\lfloor\mathbf{f} N^{d}\right\rfloor}} Z(\beta, z, V)
$$


is obtained by summing over all $N$-boxes. So we can proceed as in the previous section, introducing the auxiliary non-interacting $\mathbf{y}$ particles, filling the complement $\mathbb{T}_{N}^{d} \backslash V$ and having the activity $\zeta=\zeta(z, \beta)$ which brings them into equilibrium with the $\mathbf{x}$-field, and try to apply the Wulff construction in this situation.

\subsection{Surface tension: conjectures}

The first thing to be done is the definition of the surface tension. Contrary to the Ising model case, which has one Gibbs state once $h>0$, here the situation is different, and it is reasonable to expect that when both $\beta$ and $z$ are large, our models have continuum of extremal Gibbs states. In the 3D case one expects the breaking of both the rotation and translation symmetry, while in the $2 \mathrm{D}$ case the translation symmetry is not broken, [Ri], and only rotation symmetry is expected to be broken. Therefore the definition of the surface tension should include the choice of the pure phase. For the hard core models defined above we take for the boundary condition $\eta^{\uparrow}$ the centers of the densest lattice packing $\Pi^{d}$ of balls of radius $\frac{(1+\varepsilon(\beta, z, d))}{2}$ in $\mathbb{R}^{d}$, with one ball centered at the origin. The orientation of the lattice is chosen in such a way that the intersection of the packing $\Pi^{d}$ with the horizontal plane $\mathbb{R}^{d-1} \subset \mathbb{R}^{d}$ results in the packing $\Pi^{d-1}$. The parameter $\varepsilon(\beta, z, d)$ is chosen in such a way that the density of points in the configuration $\eta^{\uparrow}$ coincides with the density of particles in a Gibbs state defined by the weight (9) . In particular, $\varepsilon(\beta, z, d) \rightarrow 0$ if $\beta \rightarrow \infty$ or if $z \rightarrow \infty$. 1

Similarly to the Section 2.2 .2 , for every $\theta \in \mathbb{S}^{d-1}$ we introduce the point configuration $\eta^{\uparrow, \theta}$, which coincides with $\eta^{\uparrow}$ in the half-space $\mathbb{R}_{\theta}=\left\{x \in \mathbb{R}^{d}:\langle x, \theta\rangle \geq 0\right\}$ and which is empty in the remaining half-space. Then we consider the partition functions $Z\left(\beta, z, \zeta(z, \beta), B_{n} ; \eta^{\uparrow, \theta}\right), Z\left(\beta, z, \zeta(z, \beta), B_{n} ; \eta^{\uparrow}\right)$, and $Z\left(\beta, z, \zeta(z, \beta), B_{n} ; \varnothing\right)$ in the cubic box $B_{n}$, and we define

$\tau_{\beta, z}^{\uparrow}(\theta)=\lim _{n \rightarrow \infty}-\frac{1}{\beta l(\theta, n)} \ln \frac{Z\left(\beta, z, \zeta(z, \beta), B_{n} ; \eta^{\uparrow, \theta}\right)}{\sqrt{Z\left(\beta, z, \zeta(z, \beta), B_{n} ; \eta^{\uparrow}\right) Z\left(\beta, z, \zeta(z, \beta), B_{n} ; \varnothing\right)}}$,

where, again, $l(\theta, n)$ is the measure of intersection of the cube $B_{n}$ with the plane $\langle x, \theta\rangle=0$. At present, there is

\footnotetext{
${ }^{1}$ In the initial version of the present paper the parameter $\varepsilon(\beta, z, d)$ was absent. The idea to introduce it is due to T. Richthammer.
} 
1. no proof of existence of the limit function $\tau_{\beta, z}^{\uparrow}(\theta)$,

2. no proof of positivity and non-trivial dependence of $\tau_{\beta, z}^{\uparrow}(\theta)$ on $\theta$ for large $\beta$ and $z$.

If we assume both, then it is safe to conjecture that the analog of the theorem 1 holds in the present situation, with the Wulff shape $W(\beta, h)$ replaced by $W^{\uparrow}(\beta, z)$, which is the solution of the Wulff problem corresponding to

the surface tension $\tau_{\beta, z}^{\uparrow}$. However, there is an important difference: in the relation (5), instead of the shift $\Gamma+x(\Gamma)$ of the crystal $\Gamma$ one has to consider also the rotated crystal, $\rho(\Gamma) \circ[\Gamma+x(\Gamma)]$, where $\rho(\Gamma) \in S O(d)$. This extra rotation appears due to the choice of one of many possible low temperature phases of our model, made in (10).

It seems that the simplest interaction for which the above conjectures $\mathbf{1}$ and 2 can be proven in all dimensions $d \geq 2$ is the one given by (요 .

\section{High temperature}

At high temperature (and low activity) we find ourselves in the uniqueness regime, while the surface tension vanishes. As a result, no large crystal is formed, i.e. all droplets are small. In the example of water bubble in oil it means that water will be dispersed into infinitesimal droplets of no specific shape.

Acknowledgement. I thank the Department of Mathematics of the University of Texas at Austin for its hospitality during my visit in May, 2017. I thank Professor Ch. Radin for the enlightening discussions of topics treated in this paper.

\section{References}

[B] Bodineau, T. (1999). The Wulff construction in three and more dimensions. Comm. Math. Phys. 207 197-229.

[BIV] T.Bodineau, D.Ioffe, Y.Velenik: Rigorous Probabilistic Analysis of Equilibrium Crystal Shapes, J. Math. Phys., Vol. 41, Nr. 3 (2000), p. 1033-1098 
[CP] Cerf, R. and Pisztora, A. (2000). On the Wulff crystal in the Ising model. Ann. Probab. 28 947-1017.

[DKS] R.L. Dobrushin, R. Kotecky and S. B. Shlosman: Wulff construction: a global shape from local interaction, AMS translations series, Providence (Rhode Island), 1992.

[K] Kenyon, Richard: Dominos and the Gaussian free field; Annals of probability (2001): 1128-1137.

[R] Charles Radin (2016) Wulff shape for equilibrium phases, arXiv:1610.08564v2

[Ri] Richthammer, T. Lower Bound on the Mean Square Displacement of Particles in the Hard Disk Model, Commun. Math. Phys. (2016) 345: 1077.

[S] Ya. G. Sinai: Theory of phase transitions: Rigorous results, Pergamon Press, Oxford-New York-..., 1982.

[SS] R. H. Schonmann and S. Shlosman: Wulff droplets and the metastable relaxation of the kinetic Ising models, Comm. Math. Phys., 194, 389462, 1998. 